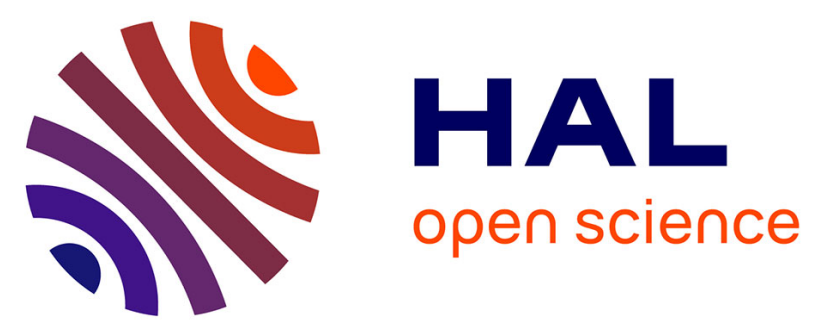

\title{
An LMI Approach for Stability Analysis and Output-Feedback Stabilization of Discrete-Time Lur'e Systems Using Zames-Falb Multipliers
}

Ariádne L J Bertolin, Ricardo C L F Oliveira, Giorgio Valmorbida, Pedro L D

Peres

\section{To cite this version:}

Ariádne L J Bertolin, Ricardo C L F Oliveira, Giorgio Valmorbida, Pedro L D Peres. An LMI Approach for Stability Analysis and Output-Feedback Stabilization of Discrete-Time Lur'e Systems Using ZamesFalb Multipliers. IEEE Control Systems Letters, 2021, 6, pp.710-715. 10.1109/LCSYS.2021.3086427 . hal-03485392

\section{HAL Id: hal-03485392 \\ https://hal.science/hal-03485392}

Submitted on 17 Dec 2021

HAL is a multi-disciplinary open access archive for the deposit and dissemination of scientific research documents, whether they are published or not. The documents may come from teaching and research institutions in France or abroad, or from public or private research centers.
L'archive ouverte pluridisciplinaire $\mathbf{H A L}$, est destinée au dépôt et à la diffusion de documents scientifiques de niveau recherche, publiés ou non, émanant des établissements d'enseignement et de recherche français ou étrangers, des laboratoires publics ou privés. 


\title{
An LMI approach for stability analysis and output-feedback stabilization of discrete-time Lur'e systems using Zames-Falb multipliers
}

\author{
Ariádne L. J. Bertolin, Ricardo C. L. F. Oliveira, Giorgio Valmorbida, Pedro L. D. Peres
}

\begin{abstract}
This paper investigates the problem of stability analysis and output-feedback stabilization of discrete-time Lur'e systems where the nonlinearity is odd and slope bounded. Using the linear matrix inequality (LMI) conditions from the literature to handle the $\ell_{1}$ norm and positive realness constraints, an iterative algorithm based on LMIs is constructed to assess stability through the existence of a Zames-Falb multiplier of any given order based on independent positive definite matrices for the $\ell_{1}$ norm and positive realness. More important, the method can also deal with output-feedback stabilization. Numerical examples illustrate the performance of the proposed approach when compared with other methods.
\end{abstract}

\section{INTRODUCTION}

The feedback interconnection of a single-input singleoutput linear time invariant (LTI) system $G$ and a static nonlinear function $\phi$, as illustrated in Figure 1, is known as a Lur'e system.

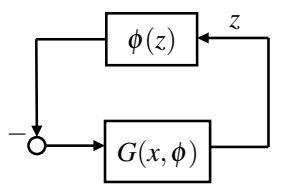

Fig. 1. Block diagram of a Lur'e system.

The stability analysis of this interconnection, the absolute stability problem, is a nonlinear robust stability problem, since the analysis is carried out for classes of nonlinearities. The most general results consider sector nonlinearities, namely nonlinearities satisfying $\phi(0)=0$ and, $\forall z \in \mathbb{R} \backslash$ $\{0\}, \underline{\alpha} \leq \phi(z) / z \leq \bar{\alpha}$. Classical results such as the Circle and Popov criteria in continuous-time assume only sector boundedness of the nonlinearity. In discrete-time, however, slope restriction of the nonlinearity is assumed since the first results [1]-[5].

Slope restriction means that $\forall z_{1}, z_{2} \in \mathbb{R}, z_{1} \neq z_{2}$, one has $\underline{\beta} \leq\left(\phi\left(z_{1}\right)-\phi\left(z_{2}\right)\right) /\left(z_{1}-z_{2}\right) \leq \bar{\beta}$. Clearly, slope restriction

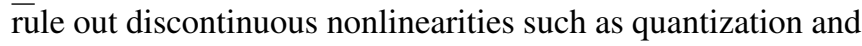
relay functions. On the other hand, sector- and slope-bounded

Supported by the Brazilian agencies Coordenação de Aperfeiçoamento de Pessoal de Nível Superior (CAPES) - Finance Code 001, CNPq and grants 2019/10947-1, São Paulo Research Foundation (FAPESP).

A. L. J. Bertolin, P. L. D. Peres and R. C. L. F. Oliveira are with the School of Electrical and Computer Engineering, University of Campinas - UNICAMP, 13083-852, Campinas, SP, Brazil. \{ariberto, ricfow, peres\}@dt. fee.unicamp.br.

G. Valmorbida is with the Laboratoire des Signaux et Systèmes, CentraleSupélec, CNRS, University of Paris-Sud, Université Paris-Saclay, Gifsur-Yvette 91192, France. He is also with Inria projet DISCO. (e-mail: giorgio.valmorbidall2s. centralesupelec.fr). nonlinearities still appear in a large class of engineering problems, modeling continuous piece-wise affine nonlinearities, such as saturating actuator and also smooth nonlinearities.

The solution to the absolute stability problem is a certificate obtained only using the LTI system data, the sector and slope bounds. There are two methods, using different representations of the LTI system: in the time-domain, with Lyapunov functions working as certificates, or in the frequency domain (within an input-output framework), where the stability certificates are transfer functions known as multipliers. The rich literature on the topic keeps growing, and different aspects, such as local stability analysis, have recently been exploited [6], [7]. In particular, the absolute stability problem has benefited from constructive approaches, based on convex optimization [8], [9].

In the input-output framework, the stability analysis is performed via loop transformations, where the multipliers, are added to the feedback connection (e.g., the multiplier to the nonlinear and its inverse to the linear branch). The problem is to search for multipliers that preserve the properties of the nonlinearity for the nonlinear branch and assure stability. In this context, a fundamental result to characterize multipliers was given by O'Shea for continuous-time systems and slope-restricted and monotone (i.e., $\beta=0$ ) nonlinearities. See the tutorial paper [10] for details. Those multipliers, called Zames-Falb (ZF) multipliers, preserve the positivity of the input-output relation of the nonlinear branch, by imposing a constraint on the $\mathscr{L}_{1}$-norm of the multiplier impulse response. The counterpart for discrete-time systems has been proposed in [11], [12, Chapter 6]. Unfortunately, the numerical search for a stability certificate in the form of a multiplier is difficult, since the discrete-time ZF multipliers have to verify an $\ell_{1}$ norm constraint. Parameterizations allowing for a convex search of a class of ZF multipliers were first proposed for continuous-time systems [13]. Concerning the discrete-time case, ZF multipliers obtained from the solution of semidefinite programming can be found in [14] or, more recently, in [15], [16]. In particular, [15] proposed finite-impulse response (FIR) ZF multipliers and [16] allows for the search of noncausal ZF multipliers.

Even though significant progress has been achieved in the stability analysis in recent years, the design of feedback control laws has received less attention. In this context, only a few results allowing to construct feedback laws for Lur'e systems were proposed. These results consider the time-domain approaches and rely on quadratic Lyapunov functions. The use of ZF multipliers for the synthesis problems, to the best 
of the authors' knowledge, has not been studied and the joint search for multipliers and control gains can benefit from the conservatism reduction obtained by the ZF multipliers.

The aim with this paper is to propose a synthesis procedure based on LMIs for nonlinear output feedback stabilization of discrete-time Lur'e systems. As a novelty, control gains are directly variables of the problem and, thanks to an appropriate relaxation procedure, the synthesis conditions can be solved by means of an iterative algorithm with local convergence. The stabilizability conditions rely on constraints for the multipliers as in [14], [15]. The novelty is the possibility of constructing multipliers of any given order to assess the $\ell_{1}$ norm and the real positiveness constraints. As demonstrated by numerical examples, the method can be less conservative than the method in [14] and is suitable for the computation of feedback gains.

Notation: For a symmetric matrix, $A>0(A<0)$ means that $A$ is positive (negative) definite. For matrices or vectors $\left(^{\top}\right)$ indicates the transpose and $\operatorname{He}(A)=A+A^{\top}$. The symbol $\star$ represents a symmetric term in a blocked square matrix and $\operatorname{diag}\left(A_{1}, A_{2}\right)$ denotes a block diagonal matrix composed by blocks $A_{1}$ and $A_{2}$. The identity matrix and the zero matrix are denoted, respectively, by $I$ and 0 . The symbol $\mathbb{1}_{c}$ represents a vector of dimension $c$ with 1 in all positions.

\section{PROBLEM DEFINITION AND PRELIMINARIES}

The stability problem is presented in what follows. The extension to cope with control design is briefly discussed in Section IV. Consider the discrete-time nonlinear Lur'e system with state-space representation given by

$$
\begin{aligned}
x(k+1) & =A x(k)+B_{\phi} \phi(z(k)) \\
z(k) & =C_{z} x(k)+D_{\phi} \phi(z(k)), \quad k \in \mathbb{Z}, k \geq 0
\end{aligned}
$$

where $x \in \mathbb{R}^{n_{x}}$ is the state, $\phi \in \mathbb{R}$ is the nonlinear input and $z \in \mathbb{R}$ is the output. Matrices $A, B_{\phi}, C_{z}$ and $D_{\phi}$ are real and have appropriate dimensions. The time-invariant nonlinearity $\phi: \mathbb{R} \rightarrow \mathbb{R}, \phi(0)=0$, is an odd monotone function that verifies the slope bound condition given by

$$
0 \leq \frac{\phi(\hat{z})-\phi(z)}{(\hat{z}-z)} \leq \Lambda
$$

for all $z, \hat{z} \in \mathbb{R}, \quad z \neq \hat{z}$, and $\phi(\hat{z}), \phi(z) \in[0, \Lambda]$ where $\Lambda \in \mathbb{R}$ is a given positive scalar. Furthermore, it is assumed that system (1) also satisfies the following wellposedness condition $\left(1+\Lambda D_{\phi}\right)>0$. By performing a loop transformation, the stability of the interconnection illustrated in Figure 1 of $G$ (transfer function associated with the quadruple $\left.\left(A, B_{\phi}, C_{z}, D_{\phi}\right)\right)$ and $\phi$ can be investigated through the existence of a ZF [17] multiplier $M(z)=1-H(z)$, such that

$$
\operatorname{Re}\left\{M\left(e^{j \omega}\right) \tilde{G}\left(e^{j \omega}\right)\right\}>0, \forall \omega \in[0,2 \pi]
$$

is verified, with $\tilde{G}\left(e^{j \omega}\right)=\left(1+\Lambda G\left(e^{j \omega}\right)\right)$ and $H(z)$ a rational strictly proper transfer function. A state-space realization for the multiplier $M(z)$ is given by

$$
\begin{gathered}
x_{m}(k+1)=A_{m} x_{m}(k)+B_{m} r(k) \\
y_{m}(k)=C_{m} x_{m}(k)+r(k),
\end{gathered}
$$

where $x_{m} \in \mathbb{R}^{n_{M}}$ is the state, $r \in \mathbb{R}$ is the input and $y_{m} \in \mathbb{R}$ is the output, with matrices $A_{m}, B_{m}$, and $C_{m}$ of appropriate dimensions. As a consequence, the state space realization of $M(z) \tilde{G}(z)$ is given by

$$
\begin{array}{cc}
A_{I}=\left[\begin{array}{cc}
A & 0 \\
B_{m} \Lambda C_{z} & A_{m}
\end{array}\right], & B_{I}=\left[\begin{array}{c}
B_{\phi} \\
B_{m}\left(1+\Lambda D_{\phi}\right)
\end{array}\right] \\
C_{I}=\left[\begin{array}{ll}
\Lambda C_{z} & C_{m}
\end{array}\right], & D_{I}=\left(1+\Lambda D_{\phi}\right) .
\end{array}
$$

Since the nonlinearities are assumed to be odd, a sufficient condition to search for the multiplier $M(z)$ assuring the stability of the loop is to test the positive realness of system $M(z) \tilde{G}(z)$ while imposing $\|H\|_{1}<1$ (thus assuring that $M(z)$ is a ZF multiplier) [14], [17]. As in [14], the method can be adapted for the design of anti-causal multipliers, by changing matrices $A, B_{\phi}, C_{z}$ and $D_{\phi}$. Defining an LTI discrete-time system $\mathscr{S}$ with state space realization $(A, B, C, D)$, LMI conditions for the computation of a bound to the $\ell_{1}$ norm and positive realness of $\mathscr{S}$ (see for instance [14]) are reproduced below.

Lemma 1 Let $\lambda \in(0,1)$ be a given real scalar. System $\mathscr{S}$ is stable with $\ell_{1}$ norm bounded by $\sqrt{\gamma}$ if there exist $\mu>0$ and a positive definite matrix $S=S^{\top}$ such that

$$
\begin{gathered}
{\left[\begin{array}{cc}
A^{\top} S A-\lambda S & A^{\top} S B \\
\star & B^{\top} S B-\mu I
\end{array}\right]<0} \\
{\left[\begin{array}{cc}
(\lambda-1) S+C^{\top} C & C^{\top} D \\
\star & (\mu-\gamma) I+D^{\top} D
\end{array}\right]<0 .}
\end{gathered}
$$

Lemma 2 System $\mathscr{S}$ is stable and positive real if there exists a positive definite matrix $P=P^{\top}$, such that

$$
\left[\begin{array}{cc}
A^{\top} P A-P & A^{\top} P B-C \\
\star & B^{\top} P B-D^{\top}-D
\end{array}\right]<0 .
$$

A new LMI condition to search for a ZF multiplier $M(z)$ assuring the stability of the interconnection is proposed in next section. As main difference from the conditions in [14], the state-space matrices associated to $M(z)$ appear affinely in the inequalities and no change of variables is necessary. Finsler's Lemma, presented next, is used for the derivation of the proposed conditions.

Lemma 3 (Finsler's lemma [18]) Consider matrices $\mathscr{Q} \in$ $\mathbb{R}^{\ell \times \ell}$ and $\mathscr{B} \in \mathbb{R}^{m \times \ell}$, with $\operatorname{rank}(\mathscr{B})<\ell$ and $\mathscr{B} \mathscr{B}_{\perp}=0$. Then, the following conditions are equivalent:

$$
\begin{aligned}
& \text { i) } \mathscr{B}_{\perp}^{\top} \mathscr{Q} \mathscr{B}_{\perp}<0 \text {; } \\
& \text { ii) } \exists \mathscr{X}^{\top} \in \mathbb{R}^{\ell \times m} \text { such that } \mathscr{Q}+\mathscr{X} \mathscr{B}+\mathscr{B}^{\top} \mathscr{X}^{\top}<0 \text {. }
\end{aligned}
$$

\section{MAIN RESULTS}

Theorem 1 Let the matrices $\bar{Y}_{i} \in \mathbb{R}^{n_{M} \times n_{M}}, i=1,3,4, \bar{Y}_{2} \in$ $\mathbb{R}^{n_{M} \times 1}, \bar{X}_{i} \in \mathbb{R}^{\left(n_{x}+n_{M}\right) \times\left(n_{x}+n_{M}\right)}, i=1,3,4, \bar{X}_{2} \in \mathbb{R}^{\left(n_{x}+n_{M}\right) \times 1}$, with $\bar{Y}_{4}$ and $\bar{X}_{4}$ of full rank, the real constant $\lambda \in(0,1)$ and the positive integer $n_{M}$ be given. If there exist matrices $Y_{i} \in \mathbb{R}^{n_{M} \times n_{M}}, i=1,3,4, \quad Y_{2} \in \mathbb{R}^{1 \times n_{M}} X_{i} \in \mathbb{R}^{\left(n_{x}+n_{M}\right) \times\left(n_{x}+n_{M}\right)}$, $i=1,3,4, X_{2} \in \mathbb{R}^{1 \times\left(n_{x}+n_{M}\right)}, 0<P^{\top}=P \in \mathbb{R}^{\left(n_{x}+n_{M}\right) \times\left(n_{x}+n_{M}\right)}$, 
$0<S^{\top}=S \in \mathbb{R}^{n_{M} \times n_{M}}, A_{m} \in \mathbb{R}^{n_{M} \times n_{M}}, B_{m} \in \mathbb{R}^{n_{M} \times 1}, C_{m} \in$ $\mathbb{R}^{1 \times n_{M}}, \mu>0$ and $0<\gamma \leq 1$ such that

$$
\begin{gathered}
\mathscr{Q}_{1}+\operatorname{He}(\underbrace{\left[\begin{array}{l}
Y_{1} \\
Y_{2} \\
Y_{3} \\
Y_{4}
\end{array}\right]}_{Y} \underbrace{\left.\begin{array}{llll}
\bar{Y}_{1} & \bar{Y}_{2} & \bar{Y}_{3} & \bar{Y}_{4}
\end{array}\right]}_{\bar{Y}})<0, \\
{\left[\begin{array}{ccc}
(\lambda-1) S & 0 & -C_{m}^{\top} \\
\star & (\mu-\gamma) & 0 \\
\star & \star & -1
\end{array}\right]<0}
\end{gathered}
$$

and

$$
\mathscr{Q}_{2}+\operatorname{He}(\underbrace{\left[\begin{array}{l}
X_{1} \\
X_{2} \\
X_{3} \\
X_{4}
\end{array}\right]}_{X} \underbrace{\left[\begin{array}{llll}
\bar{X}_{1} & \bar{X}_{2} & \bar{X}_{3} & \bar{X}_{4}
\end{array}\right]}_{\bar{X}})<0,
$$

where

$$
\mathscr{Q}_{1}=\left[\begin{array}{cccc}
-\lambda S & 0 & 0 & A_{m}^{\top} \\
\star & -\mu & 0 & B_{m}^{\top} \\
\star & \star & S & -I \\
\star & \star & \star & 0
\end{array}\right], \mathscr{Q}_{2}=\left[\begin{array}{cccc}
-P & -C_{I}^{\top} & 0 & A_{I}^{\top} \\
\star & -2 D_{I} & 0 & B_{I}^{\top} \\
\star & \star & P & -I \\
\star & \star & \star & 0
\end{array}\right],
$$

are verified, then the Lur'e system (1) is stable with the $Z F$ multiplier realization given by $A_{m}, B_{m}$ and $C_{m}$.

Proof: Consider

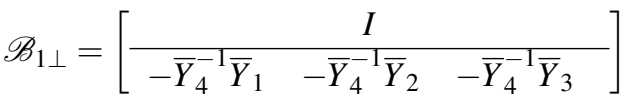

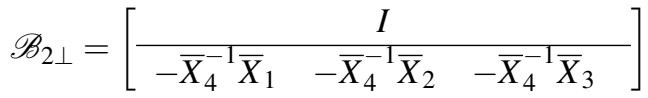

respectively as basis for the null spaces of $\bar{Y}$ and $\bar{X}$, that is, $\bar{Y} \mathscr{B}_{1 \perp}=0$ and $\bar{X} \mathscr{B}_{2 \perp}=0$. Invoking Lemma 3, (6a) is equivalent to $\mathscr{B}_{1 \perp}^{\top} \mathscr{Q}_{1} \mathscr{B}_{1 \perp}<0$, that can be rewritten as

$$
\left[\begin{array}{ccc}
-\lambda S & 0 & 0 \\
\star & -\mu & 0 \\
\star & \star & S
\end{array}\right]+\operatorname{He}\left(\left[\begin{array}{c}
\left(\bar{Y}_{4}^{-1} \bar{Y}_{1}\right)^{\top} \\
\left(\bar{Y}_{4}^{-1} \bar{Y}_{2}\right)^{\top} \\
\left(\bar{Y}_{4}^{-1} \bar{Y}_{3}\right)^{\top}
\end{array}\right]\left[\begin{array}{lll}
A_{m} & B_{m} & -I
\end{array}\right]\right)<0 .
$$

Last inequality is in the form ii) of Lemma 3 and, computing a basis for the null space of $\left[\begin{array}{lll}A_{m} & B_{m} & -I\end{array}\right]$, the following equivalent condition can be obtained

$$
\left[\begin{array}{cc}
I & 0 \\
0 & 1 \\
A_{m} & B_{m}
\end{array}\right]^{\top}\left[\begin{array}{ccc}
-\lambda S & 0 & 0 \\
\star & -\mu & 0 \\
\star & \star & S
\end{array}\right]\left[\begin{array}{cc}
I & 0 \\
0 & 1 \\
A_{m} & B_{m}
\end{array}\right]<0,
$$

which can be rewritten as

$$
\left[\begin{array}{cc}
A_{m}^{\top} S A_{m}-\lambda S & A_{m}^{\top} S B_{m} \\
\star & B_{m}^{\top} S B_{m}-\mu
\end{array}\right]<0 .
$$

On the other hand, applying a Schur complement in (6b), one has

$$
\operatorname{diag}\left((\lambda-1) S+C_{m}^{\top} C_{m},(\mu-\gamma)\right)<0
$$

Conditions (8) and (9) correspond to the ones of Lemma 1 (with the feedforward term zeroed) assuring, since $0<\gamma \leq 1$, $\|H\|_{1}<1$, i.e., $M(z)$ is a $\mathrm{ZF}$ multiplier.

Similarly, by Lemma 3 , condition (7) is equivalent to $\mathscr{B}_{2 \perp}^{\top} \mathscr{Q}_{2} \mathscr{B}_{2 \perp}<0$, that can be rewritten as

$$
\left[\begin{array}{ccc}
-P & -C_{I}^{\top} & 0 \\
\star & -2 D_{I} & 0 \\
\star & \star & P
\end{array}\right]+\operatorname{He}\left(\left[\begin{array}{l}
\left(\bar{X}_{4}^{-1} \bar{X}_{1}\right)^{\top} \\
\left(\bar{X}_{4}^{-1} \bar{X}_{2}\right)^{\top} \\
\left(\bar{X}_{4}^{-1} \bar{X}_{3}\right)^{\top}
\end{array}\right]\left[\begin{array}{lll}
A_{I} & B_{I} & -I]
\end{array}\right]<0 .\right.
$$

The above inequality is, again, in the form ii) of Lemma 3. Then, computing a basis for the null space of $\left[\begin{array}{ll}A_{I} & B_{I}-I\end{array}\right]$, the following condition, equivalent to (5), can be obtained

$$
\left[\begin{array}{ll}
A_{I}^{\top} P A_{I}-P & A_{I}^{\top} P B_{I}-C_{I}^{\top} \\
B_{I}^{\top} P A_{I}-C_{I} & B_{I}^{\top} P B_{I}-2 D_{I}
\end{array}\right]<0,
$$

proving the stability of the interconnected system.

Besides presenting the interesting fact that the multiplier $M(z)$ can be of any given order $n_{M} \geq 1$, the stability conditions of Theorem 1 also have two additional appealing features. First note that, differently from [14], two independent positive definite matrices are used, namely $P$ and $S$, one to impose $\|H\|_{1}<1$ and another to certify the positive realness of $M(z) \tilde{G}(z)$. More interestingly, note that the matrices of the system appear affinely in the conditions. This is used to develop a method for the synthesis of controllers based on the ZF approach, as discussed in Section IV.

On the other hand, the drawback of Theorem 1 is that matrices $\bar{X}_{i}$ and $\bar{Y}_{i}$ must be given. Clearly, this is a source of conservativeness. Next theorem proposes a strategy to overcome this limitation.

Theorem 2 Let $\bar{Y}=\left[\begin{array}{lll}0 & v \mathbb{1}_{n_{M}}-I I\end{array}\right]$, such that $v^{2}<1 / n_{M}$, $\bar{X}=\left[\begin{array}{llll}0 & 0 & - & I\end{array}\right]$ and the change of variables

$$
\left(\bar{A}_{m}, \bar{B}_{m}, \bar{C}_{m}\right)=\rho^{-1}\left(A_{m}, B_{m}, C_{m}\right)
$$

$$
\begin{gathered}
\bar{A}_{I}=\frac{1}{\rho} A_{I}=\left[\begin{array}{cc}
\rho^{-1} A & 0 \\
\bar{B}_{m} \Lambda C_{z} & \bar{A}_{m}
\end{array}\right], \bar{B}_{I}=\frac{1}{\rho} B_{I}=\left[\begin{array}{c}
\rho^{-1} B_{\phi} \\
\bar{B}_{m}\left(1+\Lambda D_{\phi}\right)
\end{array}\right] \\
\bar{C}_{I}=\frac{1}{\rho} C_{I}=\left[\begin{array}{ll}
\rho^{-1} \Lambda C_{z} & \bar{C}_{m}
\end{array}\right],
\end{gathered}
$$

where $\rho>0$. Then, the conditions of Theorem 1 always have a feasible solution with a finite value of $\rho$.

Proof: First, applying a Schur complement in inequality (6b), one has

$$
\operatorname{diag}\left((1-\lambda) S-\bar{C}_{m}^{\top} \bar{C}_{m},(\gamma-\mu)\right)>0
$$

that is verified with $\bar{C}_{m}=0$ (note that $\lambda \in(0,1)$ ) and $\gamma-\mu>$ 0 . In condition (6a), adopting the proposed choices and fixing $Y_{1}=0, Y_{2}=0.5 v \mathbb{1}_{n_{M}}^{\top}, \bar{A}_{m}=0$ and $\bar{B}_{m}=0$, one gets

$$
\left[\begin{array}{cccc}
-\lambda S & 0 & 0 & 0 \\
\star & -\mu+v^{2} \mathbb{1}^{\top} \mathbb{1} & v \mathbb{1}^{\top}\left(Y_{3}-0.5 I\right) & v \mathbb{1}^{\top}\left(Y_{4}+0.5 I\right) \\
\star & \star & S-\left(Y_{3}+Y_{3}^{\top}\right) & -I-Y_{4}^{\top}+Y_{3} \\
\star & \star & \star & Y_{4}+Y_{4}^{\top}
\end{array}\right]<0 .
$$


Choosing $Y_{3}=0.5 I, Y_{4}=-0.5 I$, one has

$$
\operatorname{diag}\left(-\lambda S,-\mu+v^{2} \mathbb{1}^{\top} \mathbb{1}, S-I,-I\right)<0
$$

that, since $-\mu+v^{2} \mathbb{1}^{\top} \mathbb{1}=-\mu+v^{2} n_{M}<0$ because $v^{2}<$ $1 / n_{M}$ by assumption, holds for any $S$ satisfying $0<S<I$. Furthermore, the choices made in (11b) produce

$$
\bar{A}_{I}=\left[\begin{array}{cc}
\rho^{-1} A & 0 \\
0 & 0
\end{array}\right], \quad \bar{B}_{I}=\left[\begin{array}{c}
\rho^{-1} B_{\phi} \\
0
\end{array}\right], \quad \bar{C}_{I}=\left[\begin{array}{ll}
\rho^{-1} \Lambda C_{z} & 0
\end{array}\right] .
$$

Then, in condition (7), using $X_{1}=X_{2}=0$ and $X_{3}=-X_{4}=$ $I / 2$ one has

$$
\left[\begin{array}{cccc}
-P & -\bar{C}_{I}^{\top} & 0 & \bar{A}_{I}^{\top} \\
\star & -2 D_{I} & 0 & \bar{B}_{I}^{\top} \\
\star & \star & P-I & 0 \\
\star & \star & \star & -I
\end{array}\right]<0 .
$$

By a diagonal dominance argument, a sufficiently large value of $\rho$ assures that the feasibility of the last inequality can be guaranteed by

$$
\operatorname{diag}\left(-P,-2 D_{I}, P-I,-I\right)<0 .
$$

The negativity of blocks $(1,1)$ and $(3,3)$ is assured for $0<P<$ $I$, while the well-posedness condition $\left(1+\Lambda D_{\phi}\right)=D_{I}>0$ guarantees that $(2,2)<0$ holds.

The variable $\rho$ introduced in Theorem 2 can be viewed as a relaxation in the matrices of the system, assuring a feasible solution to the modified Theorem 1 for a sufficiently large $\rho$. However, to certify the stability of the original system, the inequalities have to hold for $\rho=1$.

Remark 1 As $\rho$ appears affinely in the conditions of Theorem 1 with the modifications proposed by Theorem 2, $\rho$ can be minimized when solving the inequalities of Theorem 1. Moreover, from a feasible solution for Theorem 1 (with some value of $\rho$ ), the resulting matrices $X_{i}^{\top}$ and $Y_{i}^{\top}$ can be used as new values for $\bar{X}_{i}$ and $\bar{Y}_{i}$, thus yielding a new feasible solution.

These features allow the formulation of an iterative procedure, Algorithm 1 (below), where $i_{\text {max }}$ sets a stop criterion and $\bar{Y}$ and $\bar{X}$ are the initial matrices required by Theorem 1 . Applying the results of Theorem 2, the procedure converges to a finite vale of $\rho$. If $\rho \leq 1$ at some iteration, then the stability of the Lur'e system can be certified. Algorithm 1 is executed for a set of values linearly spaced of $\lambda \in(0,1)$ and a fixed $n_{M} \geq 1$ (order of the multiplier $M(z)$ ).

Next theorem shows an important property of Algorithm 1.

Theorem 3 The sequence of values $\rho_{\kappa}$ provided by Algorithm 1 is non increasing.

Proof: Suppose that at iteration $\kappa$ a finite value for $\rho_{\kappa}$, associated to the variables $P_{\kappa}, S_{\kappa}, Y_{\kappa}$ and $X_{\kappa}$, has been obtained. Since $\operatorname{He}(Y \bar{Y})=\operatorname{He}\left(\bar{Y}^{\top} Y^{\top}\right)$ and $\operatorname{He}(X \bar{X})=$ $\mathrm{He}\left(\bar{X}^{\top} X^{\top}\right)$, the choices $\bar{Y}_{\kappa+1}=Y_{\kappa}$ and $\bar{X}_{\kappa+1}=X_{\kappa}$ guarantee a new feasible solution by construction, assuring that $\rho_{\kappa+1}$ cannot increase, that is, $\rho_{\kappa+1} \leq \rho_{\kappa}$.

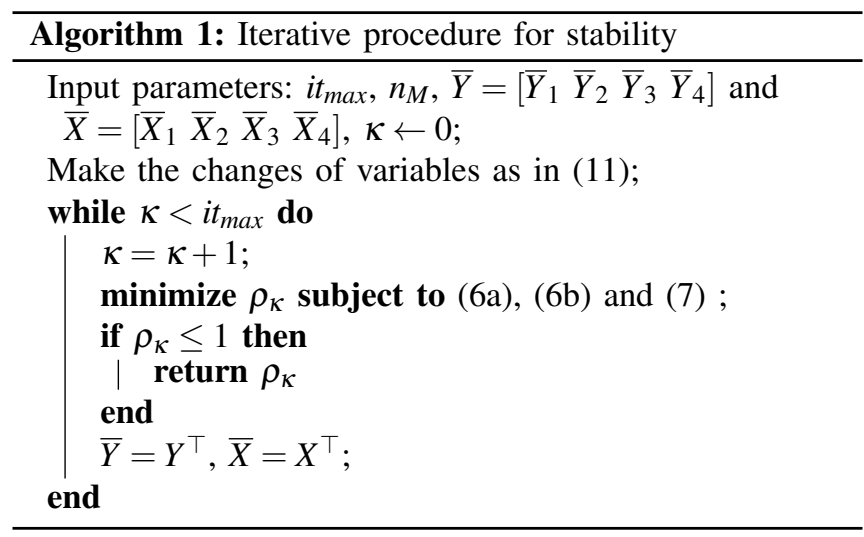

As mentioned above, the stability of the Lur'e system (1) is proven with $\rho=1$. The next theorem shows that a feasible solution with a $\rho<1$ also guarantees stability.

Theorem 4 Consider the relaxations given in Theorem 2 and the existence of two solutions of (6) and (7), at iterations $\kappa$ and $\kappa+1$, provided by Algorithm 1 such that

$$
\begin{aligned}
& \mathscr{Q}_{1, \kappa}+\operatorname{He}\left(Y_{\kappa} Y_{\kappa-1}^{\top}\right)<0, \quad \mathscr{Q}_{2, \kappa}+\operatorname{He}\left(X_{\kappa} X_{\kappa-1}^{\top}\right)<0 \\
& {\left[\begin{array}{ccc}
(\lambda-1) S_{\kappa} & 0 & -C_{m}^{\top} / \rho_{\kappa} \\
\star & \left(\mu_{\kappa}-1\right) & 0 \\
\star & \star & -I
\end{array}\right]<0, \rho_{\kappa}>1} \\
& \mathscr{Q}_{1, \kappa+1}+\operatorname{He}\left(Y_{\kappa+1} Y_{\kappa}^{\top}\right)<0, \quad \mathscr{Q}_{2, \kappa+1}+\operatorname{He}\left(X_{\kappa+1} X_{\kappa}^{\top}\right)<0 \\
& {\left[\begin{array}{ccc}
(\lambda-1) S_{\kappa+1} & 0 & -C_{m}^{\top} / \rho_{\kappa+1} \\
\star & \left(\mu_{\kappa+1}-1\right) & 0 \\
\star & \star & -I
\end{array}\right]<0, \rho_{\kappa+1}<1}
\end{aligned}
$$

Then, matrices $A_{m}, B_{m}$ and $C_{m}$ can be obtained for the original system (i.e., for $\rho=1$ ) from a convex combination of the two solutions.

Proof: Consider positive scalars $\zeta_{\kappa}$ and $\zeta_{\kappa+1}$ such that $\zeta_{\kappa}+\zeta_{\kappa+1}=1, \zeta_{\kappa}=\frac{\rho_{\kappa}\left(1-\rho_{\kappa+1}\right)}{\rho_{\kappa}-\rho_{\kappa+1}}, \zeta_{\kappa+1}=\frac{\rho_{\kappa+1}\left(\rho_{\kappa}-1\right)}{\rho_{\kappa}-\rho_{\kappa+1}}$.

Multiply the inequalities in (12) by $\zeta_{\kappa}$, (13) by $\zeta_{\kappa+1}$, summing up accordingly. Then, by analyzing $\bar{A}_{m}$ in block $(4,1)$ of the term $\zeta_{\kappa} \mathscr{Q}_{1, \kappa}+\zeta_{\kappa+1} \mathscr{Q}_{1, \kappa+1}$ one has

$$
\begin{aligned}
\zeta_{\kappa} \frac{1}{\rho_{\kappa}} A_{m, \kappa}+\zeta_{\kappa+1} \frac{1}{\rho_{\kappa+1}} A_{m, \kappa+1} & =\frac{\rho_{\kappa}\left(1-\rho_{\kappa+1}\right)}{\rho_{\kappa}-\rho_{\kappa+1}} \frac{1}{\rho_{\kappa}} A_{m, \kappa} \\
+\frac{\rho_{\kappa+1}\left(\rho_{\kappa}-1\right)}{\rho_{\kappa}-\rho_{\kappa+1}} \frac{1}{\rho_{\kappa+1}} A_{m, \kappa+1} & =\frac{1-\rho_{\kappa+1}}{\rho_{\kappa}-\rho_{\kappa+1}} A_{m, \kappa} \\
& +\frac{\rho_{\kappa}-1}{\rho_{\kappa}-\rho_{\kappa+1}} A_{m, \kappa+1}
\end{aligned}
$$

Following similar steps, $\bar{B}_{m}$ and $\bar{C}_{m}$ can also be recovered. Therefore, the stability of the original system (i.e., when $\rho=$ 1) can be assured by the ZF multiplier with matrices $A_{m}, B_{m}$ and $C_{m}$ obtained as convex combinations of the matrices that solve (12) and (13). 
To illustrate, consider that system (1), modified according to Theorem 2, is represented by $G_{\kappa}=$ $\left(A_{\kappa} / \rho_{\kappa}, B_{\phi_{\kappa}} / \rho_{\kappa}, C_{z_{\kappa}} / \rho_{\kappa}, D_{\phi}\right)$ at iteration $\kappa$. Figure 2 shows a sequence of "relaxed" systems $G_{\kappa}$ produced by Algorithm 1 until iteration $\kappa^{\star}$, where the value of the relaxation level is less than one. Note that two consecutive relaxed systems, for instance, $G_{1}$ and $G_{2}$, only have in common the set of slack variables $\bar{X}_{i 1}, X_{i 2}$ and $\bar{Y}_{i 1}, Y_{i 2}$. The positive definite matrices $P_{1}, P_{2}, S_{1}$ and $S_{2}$, and the ZF multipliers $M_{1}(z)$ and $M_{2}(z)$ are distinct. However, any system obtained as a convex combination of $G_{1}$ and $G_{2}$, that is, $\zeta G_{1}+(1-\zeta) G_{2}, \zeta \in[0,1]$, has its own multiplier by simply combining accordingly $M_{1}(z)$ and $M_{2}(z)$. This property is obtained thanks to the fact that the system matrices as well as the multipliers appear affinely in the conditions of Theorem 1. Clearly, the interest relies on the last two iterations, where a multiplier proving the stability of the original system (i.e., for $\rho=1$ ) can be extracted.

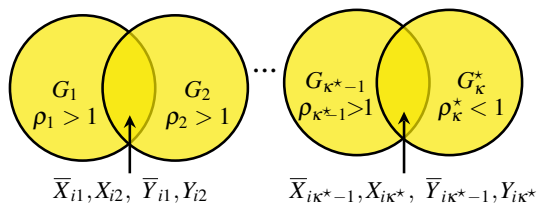

Fig. 2. Diagram illustrating the sequence of "relaxed" systems $G_{\kappa}=$ $\left(A_{\kappa} / \rho_{\kappa}, B_{\phi_{\kappa}} / \rho_{\kappa}, C_{z_{\kappa}} / \rho_{\kappa}, D_{\phi}\right)$ produced by Algorithm $1, \kappa=1, \ldots, \kappa^{\star}$.

Although the sequence of values for $\rho$ is non-increasing, as shown in Theorem 3, it is not possible to conclude on the strict decrease of $\rho$ as a function of $\kappa$. The problem (even in the context of stability analysis only) is nonconvex and, therefore, $\rho$ may converge to a value greater than one.

\section{NONLINEAR FEEDBACK CONTROL LAW}

The problem of synthesis of stabilizing controllers for Lur'e systems is investigated in this section. To the best of the authors' knowledge, this challenging problem has not been investigated before using the ZF multiplier approach in terms of LMIs. Consider system (1) with an extra control signal $u \in \mathbb{R}^{m}$ entering through matrix $B_{u}$, and a measured output $y \in \mathbb{R}^{p}, y=C_{y} x$. Under the static output-feedback control law $u=K y+K_{\phi} \phi(z)$, the state-space representation for the closed-loop system is given by

$$
\begin{aligned}
x(k+1) & =A_{c l} x(k)+B_{c l} \phi(z(k)) \\
z(k) & =C_{z} x(k)+D_{\phi} \phi(z(k))
\end{aligned}
$$

with $A_{c l}=A+B_{u} K C_{y}$ and $B_{c l}=B_{\phi}+B_{u} K_{\phi}$. As the main advantage of the proposed method when compared to previous approaches from the literature, the extension to cope with feedback design is immediate, since matrices $A_{c l}, B_{c l}, C_{z}$ and $D_{\phi}$ appear affinely in the conditions. Due to the relaxation variable $\rho$, the only setting that must be done before applying Algorithm 1 is to consider the change of variables $\bar{K}=K / \rho$ and $\bar{K}_{\phi}=K_{\phi} / \rho$.

\section{EXAMPLES}

In this section, numerical examples illustrate the performance of the proposed conditions when compared with methods from the literature. For each model, the aim (performance criterion) is to find the maximum value of $\Lambda$ such that the system is stable (or stabilizable), and this search is performed by a bisection procedure with precision given by $10^{-3}$. The LMI conditions were programmed and solved in Matlab using the parser Yalmip [19] and the solver Mosek [20]. The setup of the PC is: Core i7, 16GB RAM, Windows 10 64 bits, Matlab 8.5 (R2015a) 64 bits, Mosek 9.2.35.

\section{Experiment 1 - Stability Analysis}

Consider the systems in [15] (numbered from 1 to 6) and model 7 given by $G_{7}(z)=\left(0.5 z^{2}+0.85 z+0.598\right) /\left(z^{2}-\right.$ $0.3 z-0.46)$ with the respective state-space realizations in canonical controllable form. Algorithm 1 (A1), with $n_{M}=$ $\{1,2\}, v=0.7$ and $i t_{\max }=50$, and the conditions of [14] (ACH13) and [15] (CHZAW20), with $n_{f}=n_{b}=n^{*}$, are compared in terms of the maximum value of $\Lambda$ obtained for each system. The results are presented in Table I. The subindices $c$ and $a c$ associated to $\mathrm{A} 1$ and $\mathrm{ACH} 13$ indicate causal and anti-causal multipliers (obtained with the change of variables indicated in [14]), respectively. Note that, compared with ACH13 (also based on $\ell_{1}$ norm and positive realness), A1 provided slightly superior performance in all systems, with the order of multiplier lower than the order of the system $\left(n_{M}<n_{x}\right)$. Larger values of $n_{M}$ than the ones reported in Table I did not result in significant improvements. In general, the FIR approach CHZAW20 outperforms all the methods. Regarding the numerical complexity, A1 is more time consuming due to the need of performing iterations (which number increases as the bisection procedure approaches the maximum achievable value of $\Lambda$ ). Nevertheless, this can be seen as the price to be paid to have an analysis condition that can be extended for control design, as shown next.

\section{Experiment 2 - Control Design}

The purpose is to evaluate the performance of the proposed output-feedback stabilization conditions, using the same systems $(1, \ldots, 7)$ previously evaluated, with $C_{y}=C_{z}$ and the following control input matrices

$$
\begin{aligned}
& (1,6,7): B_{u}^{\top}=\left[\begin{array}{ll}
0 & 1
\end{array}\right], \quad(2,3): B_{u}^{\top}=\left[\begin{array}{llll}
0 & 1 & 0 & 0
\end{array}\right], \\
& \text { (4): } B_{u}^{\top}=\left[\begin{array}{lllll}
0 & 1 & 0 & 0 & 0
\end{array}\right], \quad(5): B_{u}^{\top}=\left[\begin{array}{lll}
0 & 1 & 0
\end{array}\right]
\end{aligned}
$$

The objective is to obtain the maximum $\Lambda$ such that the closed-loop system is stable. For simplicity, the same value of $\lambda$ obtained for the stability analysis, reported in the upper part of Table I, is used (new searches on $\lambda$ could improve the results). The results are shown at the bottom part of Table I in pairs $\Lambda_{s f}, \Lambda_{o f}$ with the values of $\Lambda_{s f}$ for stateand $\Lambda_{o f}$ for output-feedback (+100 means values larger than 100). The output-feedback gains and multipliers obtained with $\mathrm{A} 1$, associated with the largest values of $\Lambda$ (boldface in the bottom part of Table I), are given in Table II. For the sake of comparison, the results from the stabilization 
TABLE I

MAXIMUM $\Lambda$ (WITH CORRESPONDING $\lambda$ OR $n^{*}$ ) FOR SYSTEMS 1 TO 7 BY $A 1\left(i t_{\max }=50\right)$ AND ACH13, CAUSAL $c$ AND ANTI CAUSAL $a c$, CHZAW20 (FIR, WITH $n_{f}=n_{b}=n^{*}$ ) AND NYQUIST CRITERION. THE CONTROL DESIGN RESULTS ARE SHOWN AT THE BOTTOM PART.

\begin{tabular}{c|c|c|c|c|c|c|c}
\hline System & 1 & 2 & 3 & 4 & 5 & 6 & 7 \\
\hline $\mathrm{ACH} 13_{c}$ & $12.437(0.001)$ & $0.768(0.046)$ & $0.234(0.679)$ & $3.360(0.299)$ & $2.332(0.692)$ & $0.917(0.120)$ & $12.439(0.320)$ \\
$\mathrm{ACH} 13_{a c}$ & $1.499(0.870)$ & $0.481(0.460)$ & $0.305(0.021)$ & $3.236(0.653)$ & $2.447(0.100)$ & $1.050(0.035)$ & $11.238(0.905)$ \\
$\mathrm{A} 1_{c, n_{M}=1}$ & $12.983(0.001)$ & $0.512(0.044)$ & $0.195(0.728)$ & $2.723(0.298)$ & $2.321(0.498)$ & $0.925(0.451)$ & $14.314(0.460)$ \\
$\mathrm{A} 1_{c, n_{M}=2}$ & $12.982(0.001)$ & $0.773(0.045)$ & $0.215(0.350)$ & $3.360(0.300)$ & $2.446(0.499)$ & $0.929(0.146)$ & $14.888(0.600)$ \\
$\mathrm{A} 1_{a c, n_{M}=2}$ & $2.460(0.799)$ & $0.538(0.650)$ & $0.256(0.800)$ & $3.243(0.699)$ & $2.447(0.100)$ & $1.080(0.300)$ & $14.895(0.099)$ \\
\hline \hline CHZWA20 (FIR) & $13.511(17)$ & $1.105(2)$ & $0.312(3)$ & $3.824(2)$ & $2.447(1)$ & $1.086(1)$ & $14.895(1)$ \\
\hline Nyquist & 36.100 & 2.745 & 0.312 & 7.907 & 2.447 & 1.087 & 14.895 \\
\hline \multicolumn{8}{c|}{ Stability bounds: $\Lambda_{s f}, \Lambda_{o f}\left(\Lambda_{s f}\right.$ state- and $\Lambda_{o f}$ output-feedback) } \\
\hline $\mathrm{A} 1_{c, n_{M}=2}$ & $24.061, \mathbf{2 3 . 8 1 0}$ & $1.883,1.953$ & $20.625,0.317$ & $+100,7.466$ & $+100, \mathbf{2 . 5 4 1}$ & $27.930,1.147$ & $+100,+\mathbf{1 0 0}$ \\
\hline $\mathrm{A} 1_{a c, n_{M}=2}$ & $21.242,21.665$ & $2.331, \mathbf{2 . 1 0 8}$ & $12.677, \mathbf{0 . 3 2 4}$ & $+100, \mathbf{8 . 5 0 2}$ & $+100,2.541$ & $85.938, \mathbf{1 . 1 6 0}$ & $+100,+100$ \\
\hline BPOV20 (sector) & $19.992,19.997$ & $1.728,1.999$ & $19.618,0.324$ & $+100,5.282$ & $+100,2.541$ & $+100,1.165$ & $\mathrm{~N} / \mathrm{A}$ \\
\hline
\end{tabular}

TABLE II

OUTPUT-FEEDBACK GAINS $\left(K, K_{\phi}\right)$ AND ZF MULTIPLIERS $M(z)$ (FOR THE MAXIMUM VALUES OF $\Lambda$, IN BOLD, IN TABLE I) CERTIFIED BY A1 WITH $n_{M}=2$ IN EXPERIMENT 2, SYSTEMS 1-7.

\begin{tabular}{|l|c|c|}
\hline 1 & $(1.0955,0.6647)$ & $\frac{z^{2}-0.7711 z-0.0191}{z^{2}+0.0032 z}$ \\
\hline 2 & $(0.1062,0.4425)$ & $\frac{0.8233 z^{2}-2.5855 z+2.8053}{z^{2}-3.2657 z+2.8053}$ \\
\hline 3 & $(0.0771,0.3796)$ & $\frac{4.365 z^{2}-23.74 z+21.55}{z^{2}-5.071 z+21.55}$ \\
\hline 4 & $(-0.0872,0.4135)$ & $\frac{0.3569 z^{2}-3.2875 z+3.1978}{z^{2}-3.0567 z+3.1978}$ \\
\hline 5 & $(1.8679,0.3640)$ & $\frac{z^{2}+0.4707 z-0.1859}{z^{2}+0.3772 z-0.1629}$ \\
\hline 6 & $(-1.0088,-0.7425)$ & $\frac{3.7623 z^{2}+32.5453 z+41.9304}{z^{2}+11.3978 z+41.9304}$ \\
\hline 7 & $(-0.7311,-1.3567)$ & $\frac{z^{2}+0.0827 z-0.1262}{z^{2}-0.0447 z-0.3615}$ \\
\hline
\end{tabular}

method in [21] (BPOV20), based on a quadratic Lyapunov function, for system (1) with $D_{\phi}=0$ and only sector bounded nonlinearities, are detailed in the last row of Table I. With the exception of System 6, A1 sharply improves $\Lambda$ when compared with the open-loop values and the method of BPOV20.

\section{Conclusions}

An LMI-based iterative method for the stability analysis of discrete-time Lur'e systems in terms of ZF multipliers has been proposed. Competitive in terms of accuracy but more time-demanding, the main interest of the approach is the immediate extension to deal with feedback control. Future work aims to investigate extensions to handle noncausal and FIR multipliers as well as multiple-input multiple-output systems.

\section{REFERENCES}

[1] G. P. Szego, "On the absolute stability of sampled-data control systems," Proc. Natl. Acad. Sci. USA, vol. 50, no. 3, pp. 558-560, Sept. 1963.

[2] Y. Z. Tsypkin, "Frequency criteria for absolute stability of nonlinear sampled-data systems," Avtomat. i Telemekh., vol. 25, no. 3, pp. 281289, 1964

[3] E. I. Jury and B. W. Lee, "On the stability of a certain class of nonlinear sampled-data systems," IEEE Trans. Autom. Control, vol. 9, no. 1, pp. 51-61, Jan. 1964.

[4] _ , "On the absolute stability of nonlinear sample-data systems," IEEE Trans. Autom. Control, vol. 9, no. 4, pp. 551-554, Oct. 1964.

[5] _ - "A stability theory on multi-nonlinear control systems," in Proc. 3rd IFAC World Congr., vol. 28, London, UK, 1966, pp. A1-A11.
[6] M. Fetzer and C. W. Scherer, "Zames-Falb multipliers for invariance," IEEE Control Syst. Letts., vol. 1, no. 2, pp. 412-417, Oct. 2017.

[7] G. Valmorbida, R. Drummond, and S. R. Duncan, "Regional analysis of slope-restricted Lurie systems," IEEE Trans. Autom. Control, vol. 64, no. 3, pp. 1201-1208, Mar. 2019.

[8] V. Kapila and W. M. Haddad, "A multivariable extension of the Tsypkin criterion using a Lyapunov-function approach," IEEE Trans. Autom. Control, vol. 41, no. 1, pp. 149-152, Jan. 1996.

[9] P. Park and S. W. Kim, "A revisited Tsypkin criterion for discretetime nonlinear Lur'e systems with monotonic sector-restrictions," Automatica, vol. 34, no. 11, pp. 1417-1420, Nov. 1998.

[10] J. Carrasco, M. C. Turner, and W. P. Heath, "Zames-Falb multipliers for absolute stability: From O'Shea's contribution to convex searches," European J. Control, vol. 28, pp. 1-19, Mar. 2016.

[11] J. C. Willems and R. W. Brockett, "Some new rearrangement inequalities having application in stability analysis," IEEE Trans. Autom. Control, vol. 13, no. 5, pp. 539-549, Oct. 1968.

[12] J. C. Willems, The Analysis of Feedback Systems. Cambridge, MA, USA: The M.I.T. Press, 1971.

[13] M. C. Turner, M. Kerr, and I. Postlethwaite, "On the existence of stable, causal multipliers for systems with slope-restricted nonlinearities," IEEE Trans. Autom. Control, vol. 54, no. 11, pp. 2697-2702, Nov. 2009.

[14] N. S. Ahmad, J. Carrasco, and W. P. Heath, "LMI searches for discretetime Zames-Falb multipliers," in Proc. 52nd IEEE Conf. Decision Control, Florence, Italy, Dec. 2013, pp. 5258-5263.

[15] J. Carrasco, W. P. Heath, J. Zhang, N. S. Ahmad, and S. Wang, "Convex searches for discrete-time Zames-Falb multipliers," IEEE Trans. Autom. Control, vol. 65, no. 11, pp. 4538-4553, Nov. 2020.

[16] M. C. Turner and R. Drummond, "Discrete-time systems with slope restricted nonlinearities: Zames-Falb multiplier analysis using external positivity," Int. J. Robust Nonlinear Control, vol. 31, no. 6, pp. 22552273, Apr. 2021.

[17] G. Zames and P. L. Falb, "Stability conditions for systems with monotone and slope-restricted nonlinearities," SIAM J. Control, vol. 6, no. 1, pp. 89-108, 1968.

[18] M. C. de Oliveira and R. E. Skelton, "Stability tests for constrained linear systems," in Perspectives in Robust Control, ser. Lecture Notes in Control and Information Science, S. O. Reza Moheimani, Ed. New York, NY: Springer-Verlag, 2001, vol. 268, pp. 241-257.

[19] J. Löfberg, "YALMIP: A toolbox for modeling and optimization in MATLAB," in Proc. 2004 IEEE Int. Symp. on Comput. Aided Control Syst. Des., Taipei, Taiwan, Sept. 2004, pp. 284-289, http://yalmip. github.io.

[20] E. D. Andersen and K. D. Andersen, "The MOSEK interior point optimizer for linear programming: An implementation of the homogeneous algorithm," in High Performance Optimization, ser. Applied Optimization, H. Frenk, K. Roos, T. Terlaky, and S. Zhang, Eds. Springer US, 2000, vol. 33, pp. 197-232, http://www.mosek.com.

[21] A. L. J. Bertolin, P. L. D. Peres, R. C. L. F. Oliveira, and G. Valmorbida, "An LMI-based iterative algorithm for state and output feedback stabilization of discrete-time Lur'e systems," in Proc. 59th IEEE Conf. Decision Control, Jeju Island, Republic of Korea, Dec. 2020, pp. 2561-2566. 